\title{
Guest editorial: Marco Somalvico memorial issue
}

\author{
Ephraim Nissan • Giuseppina Gini • Marco Colombetti
}

Published online: 19 May 2009

(C) Springer Science + Business Media B.V. 2009

Keywords Marco Somalvico $\cdot$ Artificial intelligence $\cdot$ Milano $\cdot$ Italy

Mathematics Subject Classifications (2000) $68 \mathrm{~T} 01$ • 68T30 • 68T27 • 68T37 • 62B15 • $62 \mathrm{~F} 15 \cdot 62-09 \cdot 68 \mathrm{~T} 50 \cdot 01 \mathrm{~A} 70 \cdot 00 \mathrm{~B} 15$

\section{The articles in this special issue}

This journal issue, which pays tribute to the memory of Marco Somalvico, is part of a broader initiative, encompassing several journals, that each devoted to him a special issue. This is the result of a coordinated effort, and every different journal issue has its own specific profile. The issue of Annals of Mathematics and Artificial Intelligence you are holding only includes three articles, but they are dense, and provide an indepth treatment of their respective subjects.

Yimin Huang and Marco Valtorta develop the mathematics of an identifiability problem in causal Bayesian networks. A causal Bayesian network consists of a causal

Dr. Ephraim Nissan, Visiting scientist at the Department of Computing, Goldsmiths College of the University of London, New Cross, London, England, UK.

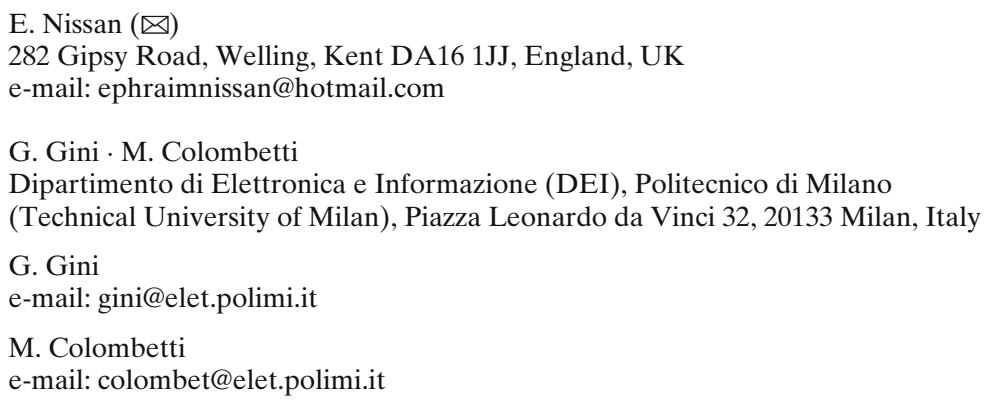


graph, an acyclic directed graph expressing causal relationships, and a probability distribution respecting the independence relation encoded by the graph. Because of the existence of unmeasured variables, the following identifiability questions arise: "Can we assess the strength of causal effects from nonexperimental data and casual relationships? And if we can, what is the total causal effect in terms of estimable quantities?" Or then, "Is it possible to compute the probability of some set of (effect) variables given intervention on another set of (intervention) variables, in the presence of non-observable (i.e., hidden or latent) variables?"

The article shows that the IDENTIFY algorithm that Tian and Pearl defined for semi-Markovian models, an important special case of causal Bayesian networks, is both sound and complete. Before this article of Huang and Valtorta, that was an important open question. Tian and Pearl combined the graphical character of causal graph and the algebraic definition of causal effect. They used both algebraic and graphical methods to identify causal effects. Tian and Pearl's method is to first transfer causal graphs to semi-Markovian graphs, and then to use the IDENTIFY algorithm to calculate the causal effects we want to know. Apart from the completeness problem, another open problem was to formally prove the equivalence of the semi-Markovian models obtained from the transforming PROJECTION algorithm, to the original causal graphs, so that therefore the causal effects should be the same in both models.

Aldo Franco Dragoni is concerned with to the inherent recursive structure of mental states. A mental structure is considered to be a potentially infinite tree, and semantics is provided for any element of that tree. The perspective is that of Belief-Desire-Intention (BDI) agents. The manner of formalizing is as a multicontext framework. Each context is a decidable theory (possibly empty) expressed in an appropriate formal language. A "mental structure" is a hierarchical lattice of triangular modules $\langle x, \mathrm{~B}, \mathrm{D}>$, where the component $x$ represents the agent $x$ 's mental state as a whole, while B and D represent specifically $x$ 's beliefs and $x$ 's desires. Based on that concept, a semantics for multi-context formalisms is provided. In Dragoni's representation of mental attitudes, these can always be represented as labelled formulae. This enables him to represent mental states as sets of labelled formulae.

We are interested in the dynamics of agent's mental states. In particular, we want to model changes of mental states after communication. For this purpose, collapsing a mental state into a single labelled formula is not much computationally appealing. This would force every speech act to change the overall mental states of the agents engaged in the communication process. It would be much better splitting that single labelled formula into syntactically smaller parts which could be handled separately in a semantically correct way. Our choice is that of grounding our dynamics of mental states on the old notion of clause.

Dragoni shows that his approach is capable of supporting "unconsciousness": rules that are "unconscious" are only those which are in the mental state's root (i.e. first level) context $x$. An interesting aspect of his representation method is that inferences themselves can be modelled as mental attitudes. Dragoni's method has, we feel, a great potential as a foundation for various kinds of development. Dragoni's paper points out that "multi-context formalism departs 
from conventional modal approaches to BDI architectures, and its semantics has little in common with classical 'Kripke-style' possible worlds one. There is no notion of 'accessibility relation' here and truth trees do not make any commitment on the interpretations of their continents. The unconstrained nature of this formalism implies that it does not make much a sense puzzling about which axioms of classical modal logic apply here since, in general, the answer is negative."

Ephraim Nissan's paper is about episodic formulae, a technique of representation for narratives and associated epistemic states he has developed and illustrated in several published journal articles. Applications range from artificial intelligence for law, to social narratives and sociocybernetics, to the mathematical humanities (from history, to literary studies). From the viewpoint of AI, promoting episodic formulae is an assertion that AI should not confine itself to problems in a closed universe, a trend that became dominant in the so-called "AI Winter" of the 1990s. Nissan's paper develops and discusses, in depth, a model for the AJIT subproject of the AURANGZEB project, which itself is typified methodologically by its combination of episodic formulae with Toulmin's argument structure (and possibly Wigmore Charts, another formalism for representing argumentation, which itself originated in legal scholarship about judicial evidence.

Another characteristic of the AURANGZEB model is its interest for modelling deception in a planning framework. Modelling deception is an active field of research in AI and multi-agent systems. An umbrella project is PLOTINUS, in which Nissan has been pursuing aspects of narrative either by means of episodic formulae, or in respect of other features (e.g., mock-explanatory tales, these being the result of a king of culture- and genre-bound abduction that is not so much an inference to the "best" explanation, but rather the generation of explanatory narratives that are driven and constrained by poetic and other conventions, even against the grain of reasoning about the real world).

As can be seen from Nissan's paper about the AJIT model in this journal issue, special care is taken to ensure that satisfactory standards are met also in terms of the humanities. Sometimes, an unexpected combination is proposed, e.g., concepts from structuralist folktale studies as being applies to a society of agents, that may be virtual avatars or even agents in a multi-robot society: this was shown in Nissan's 'Nested Beliefs, Goals, Duties, and Agents Reasoning About Their Own or Each Other's Body in the TIMUR Model: A Formalism for the Narrative of Tamerlane and the Three Painters', Journal of Intelligent and Robotic Systems, 52(3-4), pp. 515-582.

\section{The multi-journal context of the initiative}

It was six years ago, on 17 November 2002, that Prof. Marco Somalvico passed away suddenly and prematurely. Marco has never ceased to be a major influence, indeed a cherished human presence as well in one's memories, for his disciples, collaborators, and colleagues who knew him. Some of these have undertaken the initiative of several commemorative issues, hosted in a number of journals, and covering research in both artificial intelligence, and robotics. This involved a painstaking refereeing process; still, for every article in these special issues, at least one of the authors at the very least knew Marco Somalvico. He was Valtorta's and Nissan's supervisor, 
whereas Dragoni belongs to a younger generation of Italian scholars in AI, a community made possible by Somalvico's pioneering the field in Italy.

Several international scholarly journals have been participating in this memorial endeavour: the issues in the Journal of Intelligent and Fuzzy Systems, in the International Journal on Artificial Intelligence Tools, in Cybernetics and Systems, in the Journal of Intelligent and Robotic Systems, and in Applied Artificial Intelligence have already appeared. The first part of the memorial issue in Computational Intelligence is now in press, and that journal is considering a second part. A large, shared pool of referees proved extremely valuable.

Our thanks go to Martin Golumbic, the editor of the Annals of Mathematics and Artificial Intelligence, for playing host. We are also grateful to the referees who made this multi-journal initiative feasible; they are all listed alphabetically in the following (even though only part of them were involved in this particular special issue): Francesco Amigoni, Silvana Badaloni, Mirko Bordignon, Andrea Bonarini, Will Browne, Vincenzo Caglioti, Stefano Cagnoni, Riccardo Cassinis, Aldo Franco Dragoni, Susi Dulli, Efstratios (Stratis) Gallopoulos, Giuseppina Gini, Maria Gini, Moshe Goldstein, Janis Jefferies, Rodger Kibble, Jacob Kogan, Jixin Ma, Vittorio Maniezzo, Emanuele Menegatti, Richard Mitchell, Ephraim Nissan, Corrado Petrucco, Matteo Roffilli, Daniel Stamate, Jin Tian.

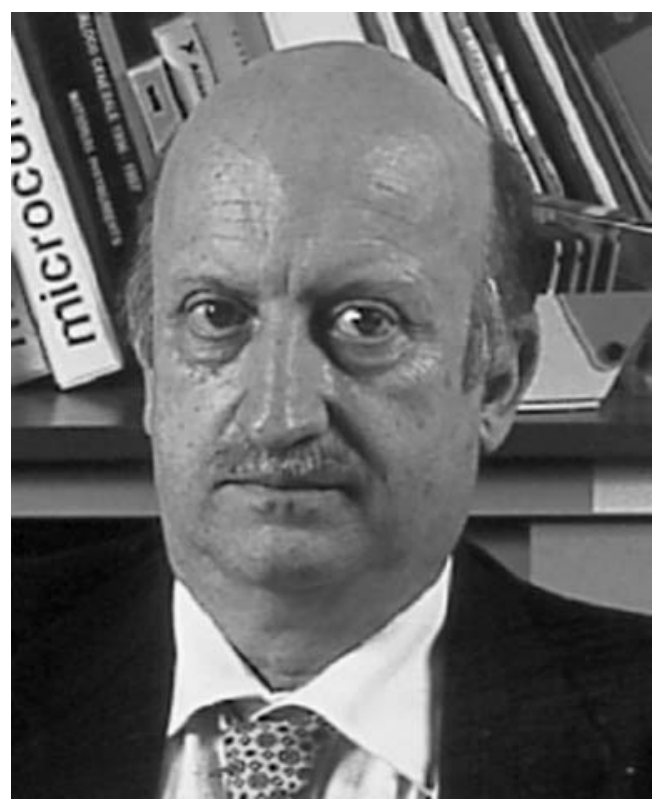

Prof. Marco Somalvico

\section{Marco Somalvico (1941-2002)}

Marco Somalvico was born in Como (the famous lakeside resort), on 10 October 1941, the son of a professional musician, and in fact he inherited a passion for music, 
being a skilled organ player. A prized high school leaver (graduate) in 1960, in the same town of Como he was to start his career as a high school teacher. In 1965, he earned the Dottore Ingegnere Degree in Electronics Engineering at the prestigious Technical University (Politecnico) of Milano, and this came along with the Medaglia D'Oro (golden medal) award of the Politecnico di Milano Alumni Association as that year's best graduate in electronics engineering. A research associateship at the Politecnico followed. After three years spent at the Artificial Intelligence Laboratory of the Computer Science Department at Stanford University (a stay which deeply influenced him), in 1971 Marco Somalvico returned to Milan, to the Computer Engineering Section of the Electronics and Information Department at Technical University, and took an assistant professor post.

In Stanford he also spent some time in 1977 and 1989, and in Edinburgh, a few months in 1972 (at the Department of Computational Logic of the School of Artificial Intelligence). As early as 1971, Marco established the Artificial Intelligence and Robotics Project of the Politecnico di Milano, where he directed the team since then. In 1974, he became associate professor locally; then, in 1980, full professor. He also was member of the Istituto di Studi Superiori dell'Insubria Gerolamo Cardano, an Institut de France-type prestigious though young institution.

At the start of the 1970s, he was the man who, at about the same time as Ugo Montanari, first introduced artificial intelligence into Italian academia. He soon brought in a robot prototype, obtained from Olivetti, which his team at the prestigious Technical University of Milan radically modified. He was among the founders of the Società Italiana di Robotica Industriale (the Tenth International Symposium of Industrial Robotics was held in Italy, in 1983), as well as of the Associazione Italiana per l'Intelligenza Artificiale. In 1987, Marco Somalvico hosted in Milan the International Joint Conference on Artificial Intelligence (IJCAI). The team carried out disparate research, with a focus on AI or robotics, but touching upon a great number of subjects. One of the projects which Marco especially cherished was applied to the assistance to people with disability, and for that reason Marco was awarded an award, the Ambrogino d'Oro, by the City of Milan. In 1998, he was awarded the Joseph F. Engelberger Robotics Award for Education (of the USA Robotic Industries Association), the equivalent of the Nobel prize for academics in robotics. These are but a few of the honours he received.

His output of scholarly publications includes five books, and over 150 articles. Apart from robotics, his research has included automated problem solving, parallel problem solving (especially using cellular automata), automated program synthesis, natural-language processing, machine vision, multi-agent systems, computer-aided design, expert systems, home and urban automation (home automation he used to call domotica in Italian), virtual museums, man-machine interaction, and AI systems of assistance to the disabled. He concerned himself as well with philosophy, and authored the entry 'Intelligenza Artificiale' for the Enciclopedia Italiana. Marco Somalvico is survived by his wife, linguist Graziella Tonfoni.

In his last several years, Marco had been constantly very tired, yet always very active. An intensely honest and deeply religious man, he also used to be combative for his convictions. There is no room to tell anecdotes about Marco's remarkable personality, blunt candour, and sometimes rather impolitic courage to stand by his conscience. He was a protagonist, and knew that. His attitude to the academic profession and to his fellow humans was very personal. He had been ill in late 2002 . 
In a phone conversation to his house in Lesmo (midway between Como and Milan), he had mentioned a recent pleural extravasation, but expected to be soon back to work. Then in the evening of 17 November, in Milan, he suffered a fatal heart failure. Italian newspapers commemorated him for the general public, at various lengths; unsurprisingly, the most showy newspaper cuts are from his native Como, whose university he co-founded. His contribution to artificial intelligence research and education was important, yet, for all of his scholarly stature, it is his personality that stands out even more. His positive personal impact on those who had the opportunity to know him and work with him was immense.

\section{The structure of the articles}

In order to facilitate going through the various topics, in the following we consider the structure of all articles by section and subsection.

On the Completeness of an Identifiability Algorithm for Semi-Markovian Models, by Yimin Huang and Marco Valtorta.

1. Introduction

2. Definitions and notations

3. Theorems and Lemmas

4. Identify Algorithm for $Q[S]$

5. Identify Algorithm for $P_{T}(S)$

6. Conclusion

References

Appendix A: Proof of Theorem 3

Appendix B: Proof of Lemma 5

Mental States as Multi-Context Systems, by Franco Aldo Dragoni

1. Introduction

1.1. The Mental Structure of an Artificial Agent

1.2. The Multi-Contextual Representation of a Mental State

2. Languages and Semantics

2.1. Languages

2.2. Semantics

3. Clausal Representation of Mental States

3.1. Mental States as Single Labelled Formulae

3.2. Clausal Representation

4. Canonical Representation and Deductive System for Mental States

5. Comments, Related and Future Work

Acknowledgments

References 


\section{Epistemic Formulae, Argument Structures, and a Narrative on Identity and Deception: A Formal Representation from the AJIT Subproject Within AURANGZEB,}

by Ephraim Nissan

\section{Introduction}

1.1. Narrative Processing, and Mathematical Formulations

1.2. Episodic Formulae

1.3. The AURANZEB Project Under the PLOTINUS Umbrella

1.4. Deception in AURANGZEB, in AI Treatments of Agents and Dialogues, in Cognitive Studies, and in Semantics

1.5. Formal Models, and Identity in Narratives

2. Toulmin Argument Structure

3. The Narrative Analysed

3.1. The Succession in Marwar after Jaswant's Death

3.2. A Clarification about Rathor Group Identity

4. The Rajput Ruse, and the Role of the Bodyguards in It

4.1. On the Role of a Bodyguard: An Antecendent in the MURAD Model

4.2. How Aurangzeb Was Outsmarted: The Exchanged Babies, and Bodyguards Sacrificing Their Lives

4.3. The Soldiers at the Orders of Aurangzeb

4.4. A Notation for Agency, Ability, Permissibility, and Communication

4.5. Eventuated or Hypothetical Relations Between Events Past or Envisaged

4.6. The Order to Seize the Two Babies and Their Mothers

4.7. A Notation for Goals

4.8. A Notation for Beliefs

4.9. A Notation for Realisation and for Hypothesis Formation

4.10. The Rathors' Counterplanning Against Aurangzeb

4.11. Remarks About Posturing and Notation

4.12. Further Details of the Counterplanning

4.13. Kinds of Perception and Testimony

4.14. Aurangzeb Would Believe the Seized Persons' Identities Was Genuine, on Being Told by His Soldiers that the Bodyguards Tried to Prevent the Outcome at the Cost of Their Lives

5. Some Formulae for Marwar's Succession Problems

5.1. Some Common Sense Knowledge About Polities

5.2. Formulae for Jaswant's Reign, and for Jagat as Being his Heir Apparent

5.3. Formulae for the Birth of the Two Posthumous Babies

6. Aurangzeb and his Vassal, Jaswant: Considerations on Being Obliged

6.1. Duties Towards a Superior Authority

6.2. The Duties of a Vassal, Obliging Behaviour, and Aurangzeb and Jaswant's Relations in the Later Period 
6.3. A Clue from Aurangzeb and Jaswant's Earlier Relations

6.4. A Fickle, Treacherous Record

6.5. Dying in the Line of Duty

6.6. Contrary-to-Duty Obligations, and Conflicting Norms

6.7. Behaving As Though Versus Make-Believe

6.8. Exchange of Favours

6.9. Overcoming Dislike for the Sake of Some Value

7. Argument Structure of Considerations on Aurangzeb's Intentions During the Marwar Interregnum

8. The Evolution of Prototypical Behaviour, and Inheritance in Taxonomic Hierarchies

9. A Matter of Different Points of View

9.1. Different Narrative Details Being Stated

9.2. On Factors Shaping Accounts: The Fate of Raja Rup Singh Rathor, vs. the Fate of Eleazar the Maccabee

9.3. Biased Reports in AI Processing of Narrative

9.4. Warping Explanation by Selectiveness and Juxtaposition

9.5. Role Reversal

10. On Analogues in Historical Narratives

11. Concluding Remarks

References

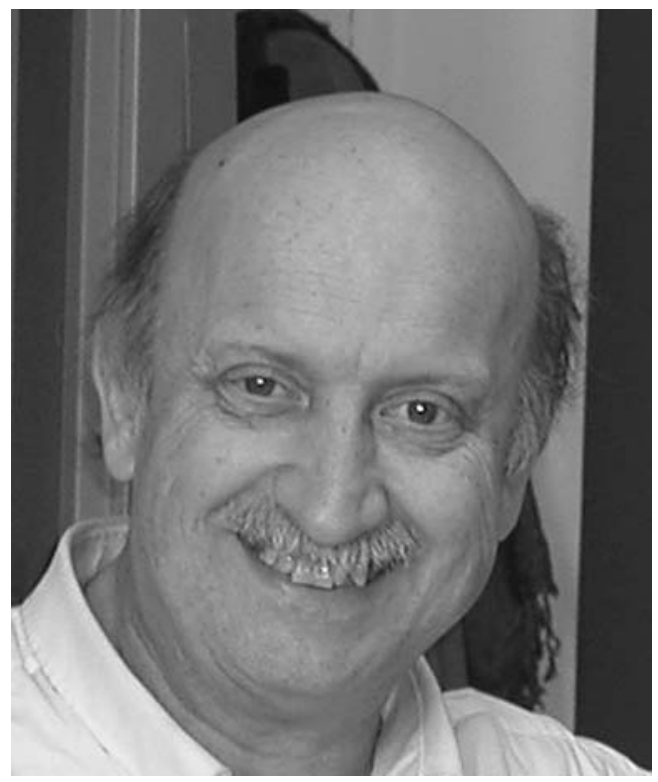

